\title{
Diferencias en la percepción de estilos parentales entre jóvenes y adultos de las mismas familias
}

\section{Differences in the paranting style pareception between parents and adolescents from the same families}

\author{
Mónica González Ramírez $\quad$ René Landero Hernández² \\ Universidad Autónoma de Nueva León, México \\ (Rec: abril de 2012 - Acep: mayo de 2012)
}

\begin{abstract}
Resumen
Se presenta una versión breve del cuestionario de prácticas parentales en formatos para padres e hijos. Los objetivos fueron: (1) proponer y evaluar las propiedades psicométricas de una versión breve del Cuestionario de Prácticas Parentales en formatos para padres y para adolescentes, y (2) comparar la percepción que tienen sobre los estilos parentales, padres y adolescentes de las mismas familias. La muestra se compone de 307 jóvenes y 197 padres, de los cuales 73 eran pares ( 73 padres y 73 adolescentes de las mismas familias). Para la reducción del cuestionario se realizó análisis factorial exploratorio y confirmatorio, así como análisis de consistencia interna, quedando una versión de 30 ítems, 15 que evalúan estilo autoritario y 15 el estilo autoritativo, con estadísticos de bondad de ajuste adecuados para el AFC y alfa de Cronbach entre .88 y .93. Para la comparación de la percepción de estilos parentales entre ambos grupos se utilizó la prueba de rangos de Wilcoxon, encontrando diferencia significativa en el estilo autoritativo, mas no en el autoritario.

Palabras clave: estilos parentales, estilo autoritativo, estilo autoritario y cuestionario de prácticas parentales.
\end{abstract}

\begin{abstract}
This paper shows a brief version of the Parenting Practices Questionnaire with forms for parents and adolescences. The objectives were (1) to evaluate psychometric properties of a brief version of the Parenting Practices Questionnaire, with forms for parents and adolescences, and (2) to compare the perception of parenting styles between parents and adolescents from the same families. 307 adolescents and 197 parents were evaluated. 73 of them were from the same families. We perform explanatory and confirmatory factor analysis as well as reliability analysis to reduce the questionnaire. The final version had 15 items to evaluate authoritative style and 15 items to evaluate authoritarian style. Goodness of fit indices were adequate and Cronbach's alpha coefficients were among .88 and .93. To compare parental style perceptions between parents and adolescents we used Wilcoxon's test, finding significant difference in authoritative style.

Keywords: parenting styles, authoritative style, authoritarian style and parenting practices questionnaire.
\end{abstract}

\footnotetext{
Correspondencia dirigida a: Mónica Teresa González Ramírez. Dr. Carlos Canseco 110. Col. Mitras Centro. C. P. 64460. Tel. (52-81) 83338233. Monterrey, Nuevo León, México.E-mail: monygzz77@yahoo.com; monica.gonzalezrz@uanl.edu.mx.

2 PhD. Profesor de la Universidad Autónoma de Nueva León, UANL, Fac. de Psicología. Av. Universidad S/N Ciudad Universitaria San Nicolás de los Garza Nuevo León, C.P. 66451 México. Tel. 83294050, e-mail: rene.landerohr@uanl.edu.mx
} 


\section{Introducción}

La teoría y la investigación sobre la familia han mostrado reiteradamente la importancia de las relaciones con los padres para el desarrollo y el ajuste psicológico de los niños y los adolescentes. Los estilos parentales de crianza vivenciados durante la niñez y la adolescencia parecen afectar el ajuste, la autoestima y la percepción de autoeficacia (Lee, Daniels y Kissinger, 2006). Durante la adolescencia temprana, el significado de las diferencias de las percepciones de padres y adolescentes en los procesos de la familia, puede ser especialmente importante, ya que las transformaciones en el desarrollo que ocurren en esta etapa, es probable que resulten en los cambios de las necesidades de los adolescentes dentro de la familia (Holmbeck, Paikoff y Brooks-Gunn, 1995).

Ser padre está relacionado con circunstancias específicas que definen y demandan una gran variedad de funciones parentales (Medina, 2004). Sin embargo, éstas están cambiando y diversificándose, con los cambios sociales ocurridos en las últimas décadas, como la incorporación de la mujer con hijos al mercado de trabajo, los cambios en la estructura y formación familiar, los demográficos y los sociopolíticos, entre otros, que han impactado en las funciones materna/ paterna, su concepción y su distribución específica en cada cultura y familia.

En palabras de Yárnoz (2006), padres y madres pueden adoptar diferentes roles con respecto a las funciones parentales. En particular en lo que se refiere a la socialización. De acuerdo a Spera (2005), el proceso de socialización se refiere a la manera por la cual un niño, a través de la educación, entrenamiento, observación y experiencia, adquiere habilidades, actitudes y conductas que son requeridas para una exitosa adaptación a una familia y cultura. No obstante, la socialización es un proceso bidireccional en el que están implicados padres e hijos, en donde los padres transmiten mensajes de socialización, pero los hijos varían en su nivel de aceptación, receptividad e internalización de esos mensajes (Grusec, Goodnow y Kuczynksi, 2000).

Para comprender mejor el proceso de socialización, es importante distinguir entre prácticas parentales y estilos parentales. Las prácticas parentales son definidas como conductas específicas que los padres usan para socializar a sus hijos. Son conductas específicas, dirigidas a un objetivo, mediante de las cuales los padres desempeñan sus obligaciones parentales (Darling y Steinberg, 1993). En cambio, estilo parental es definido como un clima emocional en el cual los padres crían a sus hijos.
Es un complejo estable de actitudes y creencias que "forman" el contexto donde ocurren las conductas paternas (Darling y Steinberg, 1993). O como señala Coloma (1993), son esquemas prácticos que reducen las múltiples y minuciosas prácticas educativas parentales a unas pocas dimensiones que, cruzadas entre sí en diferentes combinaciones, dan lugar a diversos tipos habituales de educación familiar (Coloma, 1993). El constructo estilo parental es usado para capturar las variaciones normales de los intentos de los padres para controlar y socializar a sus hijos (Baumrind, 1991).

Los estilos parentales implican prácticas parentales específicas y la combinación de éstas conformará la tipología de los estilos parentales que Baumrind (1971, 1991) propone. A los que posteriormente, con la aportación de Maccoby y Martin (1983), se agrega un cuarto estilo parental "no implicado (negligente)".

Los tres estilos parentales que Baumrind (1971, 1991) propuso son: El estilo autoritario (alto control). Son padres exigentes, pero prestan poca atención a las necesidades de sus hijos; las reglas y órdenes de estos padres no pueden ser cuestionadas ni negociadas. Se valora sobre todo la obediencia y se limita y restringe el grado de autonomía. Son muy exigentes y directivos, y esperan que sus órdenes sean obedecidas sin explicación (Baumrind, 1991).

El estilo permisivo (bajo control o dirección). Lo conforman los padres poco exigentes que atienden las necesidades de sus hijos; establecen pocas reglas de comportamiento, los consultan a la hora de tomar decisiones y son afectuosos con ellos. Los padres suelen no castigar los impulsos de sus hijos ni se ejerce prácticamente ningún control con el grado máximo de autonomía (Baumrind, 1971). Son más sensibles, no tradicionales e indulgentes, no requieren un comportamiento maduro, permiten una autorregulación considerable, y evitan la confrontación (Baumrind, 1991).

El estilo autoritativo (autoridad a través de saber, conocer y dar dirección). Son padres exigentes que atienden las necesidades de sus hijos; ellos supervisan y establecen normas claras para la conducta de sus hijos. Son asertivos, pero no intrusivos ni restrictivos. Sus métodos disciplinarios son de apoyo, más que punitivo. Ellos quieren a sus hijos asertivos y socialmente responsables, así como auto-regulados y cooperativos (Baumrind, 1971, 1991). Además los adolescentes que perciben a sus padres como autoritativos tienen un mejor ajuste psicológico y una mejor respuesta emocional, que la población con otros estilos educativos familiares (Osorio de Rebellón, Rivas, Jokin de Irala y López, 2009). 
Algunos investigadores han tratado de asociar los estilos parentales a características de personalidad. Por ejemplo, Huver, Otten, de Vries y Engels (2010) reportaron que la extroversión y empatía eran características de los padres autoritativos. Sin embargo, es importante mencionar que en la práctica educativa cotidiana, los padres ejercen dependiendo de la situación o contexto, y de lo aprendido por ellos mismos, diferentes formas de educar a los hijos. Los estilos parentales no son excluyentes, los padres pueden tener uno predominante, pero también características de los otros. Además, los padres suelen tener una percepción propia y/o diferente a la de sus hijos sobre sus prácticas parentales.

Es así que Bersabé, Fuentes y Motrico (2001), encontraron una baja concordancia entre la información aportada por los padres y sus hijos, lo que sugiere la necesidad de evaluar los estilos educativos parentales desde ambas perspectivas. Lo cual coincide con lo ya planteado por Buri (1989) y Smetana (1995), además Buri señala que la percepción de los adolescentes puede ser un importante predictor de sus respuestas, más que los reportes de los padres.

En el presente trabajo, la evaluación de los estilos parentales se ha realizado mediante cuestionarios de auto-informe y siguiendo la recomendación de Buri (1989), Smetana (1995) y Bersabé et al. (2001) de evaluar las dos perspectivas, donde los padres responden de acuerdo a su autopercepción en el rol de padres, lo mismo los adolescentes de acuerdo a su percepción como hijos. Existen diferentes opciones para evaluarlos con distintas perspectivas. Entre los que se enfocan a evaluar las prácticas parentales y siguiendo la tipología de Baumrind, se encuentran los siguientes: El de Buri (1991) que desarrolló un cuestionario de 30 ítems (PAQ) para medir los prototipos de autoridad parental propuestos por Baumrind (1971), evaluando las conductas paternas y maternas percibidas por los hijos e hijas. El cuestionario de Robinson et al. (1995), de 62 ítems, evaluando los tres estilos parentales propuestos por Baumrind; en su estudio (Robinson et al., 1996) se analiza la estructura factorial del instrumento en 4 países, comparando a ambos padres con hijos de edad preescolar.

Por otro lado, en México no se encontraron estudios o cuestionarios diseñados para adolescentes, donde los hijos manifestaran su percepción respecto al estilo parental predominante en su familia, por lo cual, hasta donde sabemos, no se ha estudiado la comparación en la percepción de estilos parentales en adolescentes y padres de las mismas familias en ese país.
Un estudio realizado en México (Gaxiola, Frías, Cuamba, Franco y Olivas, 2006), donde se utilizó el mismo cuestionario que en el presente, tuvo como objetivo medir la estructura factorial del Cuestionario de Prácticas Parentales de Robinson et al. (1995), el cual fue construido para evaluar las prácticas de los padres de acuerdo con la clasificación de Baumrind; en esa investigación se evaluó a 60 madres de familia y se llevó a cabo un análisis factorial confirmatorio. Los resultados mostraron que el instrumento evaluado presentó dos factores en los sujetos estudiados: los estilos autoritarios y autoritativos, en lugar de los tres factores propuestos por su autor.

A pesar de la limitación encontrada, respecto a que el Cuestionario de Prácticas Parentales de Robinson et al. (1995) sólo reflejaba la medición adecuada de los estilos autoritario y autoritativo, se decidió utilizar ese mismo cuestionario para poder contar con un referente en nuestro país al comparar resultados, además, la muestra empleada por Gaxiola et al. (2006) fue de un número reducido y sólo incluía madres de familia.

Es por esto que, además de buscar las diferencias en percepción de padres e hijos, se analizó para esta investigación, una versión breve del cuestionario. Asimismo, adaptamos el cuestionario para que pudiera ser respondido por los hijos y de esta manera dar respuesta a los objetivos del estudio: 1) proponer y evaluar las propiedades psicométricas de una versión breve del Cuestionario de Prácticas Parentales (PPQ) de Robinson et al. (1995), en formatos para padres y para adolescentes, y (2) comparar la percepción que tienen sobre los estilos parentales, padres y adolescentes de las mismas familias. Este segundo objetivo es el principal de la investigación, quedando el objetivo uno como un medio para alcanzar el propósito del estudio.

\section{Método}

\section{Participantes}

Se trata de un estudio no experimental, con un muestreo no probabilístico. La muestra se compone de 307 jóvenes y 197 padres, de los cuales solamente 73 son pares (padres y adolescentes de las mismas familias: 73 adultos y 73 jóvenes). Los participantes fueron evaluados en sus casas, centros de trabajo o centros de estudio. Todos viven en el área metropolitana de la ciudad de Monterrey, en el Estado de Nuevo León, México. 


\section{Instrumentos}

Como se mencionó anteriormente, se utilizó el Cuestionario de Prácticas Parentales (Parenting Practices Questionnaire) de Robinson et al. (1995) en su versión en español publicada por Gaxiola et al. (2006). El cuestionario se basa en los planteamientos teóricos de Baumrind sobre los estilos educativos de los padres. Consta de 62 ítems tipo likert, con cinco opciones de respuesta que van de nunca (1) a siempre (5) y que evalúan tres estilos parentales: autoritativo, autoritario y permisivo. Los coeficientes de consistencia interna reportados por Robinson et al. $(1995,1996)$ fueron .91, .86 y .75 , respectivamente. Estos tres estilos parentales están formados por grupos de ítems o factores, que de acuerdo a Robinson et al. (1995, 1996), determinan las prácticas parentales específicas: (1) Estilo autoritativo: a) calidez e involucramiento, b) razonamiento, c) participación democrática y d) relación amistosa (27 ítems, con un alfa de Cronbach de . 91); (2) Estilo autoritario: a) hostilidad verbal, b) castigo corporal, c) no razonamiento, estrategias punitivas y d) directividad (20 ítems, con un alfa de Cronbach de .86); (3) Estilo permisivo: a) falta de confianza en las habilidades de crianza, b) ignorar mal comportamiento y c) falta de supervisión (15 ítems, con un alfa de Cronbach de . 75).

Para la versión en español, Gaxiola et al. (2006) analizaron la estructura factorial mediante el análisis factorial confirmatorio y encontraron que el mejor modelo incluía únicamente los estilos autoritativo y autoritario, los valores de alfa de Cronbach para estos dos estilos fueron .70 y .69, respectivamente. La distribución de ítems en los factores fue: (1) Estilo autoritativo: envolvimiento, razonamiento, participación democrática, relación amistosa, falta de supervisión e ignorar mal comportamiento y (2) Estilo autoritario: hostilidad verbal, castigo corporal, no razonamiento, directividad autoritaria y falta de confianza en las habilidades de crianza.

\section{Procedimiento de análisis estadístico}

El procedimiento originalmente considerado era solamente llevar a cabo análisis de consistencia interna y análisis factorial confirmatorio, tal como lo realizaron Gaxiola et al. (2006). Sin embargo, al iniciar con el análisis de consistencia interna de los 11 grupos de ítems propuestos por Robinson et al. (1995, 1996), se encontraron valores desde .08 hasta .89 (dos arriba de .85 ; cinco entre .58 y .76 ; tres entre .08 y .37). En el estudio de Gaxiola et al. (2006) también se reportan valores inadecuados al realizar los análisis de consistencia interna con los 11 grupos de ítems. Por lo que se optó por el siguiente procedimiento, todo con el propósito de reducir la escala y proponer una versión breve.

Se inició con un análisis factorial exploratorio (AFE), con el método ejes principales rotación ortogonal (varimax) y oblicua (promax), tal como lo realizaron Robinson et al. (1996). Se probaron soluciones de 2 y 3 factores. Debido a que el método de extracción de componentes principales es el más utilizado, también se realizó el análisis factorial con este procedimiento. Para comparar las saturaciones factoriales de los métodos de extracción utilizados, se calcularon los índices de congruencia factorial (MacCallum, Widaman, Zhang y Hong, 1999), según la ecuación:

$$
V=\left[\frac{\operatorname{Trace}\left[(B-\bar{B})^{\prime}(B-\bar{B})\right]^{1 / 2}}{p r}\right]
$$

Donde:

$B$ es la matriz factorial para una determinada condición

$\bar{B} \quad$ es la matriz factorial promedio

$p \quad$ es el número de variables

$r \quad$ es el número de factores

Posteriormente se realizaron análisis de consistencia interna para cada factor. Con base en los resultados se conservó la solución bifactorial (estilos autoritario y autoritativo), seleccionando los ítems con carga factorial mayor a .40 en un sólo factor y con correspondencia teórica al estilo parental planteado (autoritario y autoritativo). Una vez reducida la escala se estimó nuevamente el AFE, utilizando tanto rotación oblicua (promax) como rotación ortogonal (varimax), mismo procedimiento empleado por Robinson et al. (1996), corroborando la estructura bifactorial.

En seguida se seleccionaron los 15 ítems para cada factor, que presentaron valores más altos en la correlación inter-ítem y en las cargas factoriales.

El cuestionario reducido a 30 ítems (15 que evalúan el estilo autoritario y 15 el autoritativo) fue sometido a AFE y análisis de consistencia interna, en la muestra de jóvenes y en la muestra de padres por separado. Finalmente, se realizó un Análisis Factorial Confirmatorio con la muestra completa $(n=504)$ para obtener los estadísticos de bondad de ajuste de la versión reducida del cuestionario.

Se tomaron en cuenta los siguientes índices de bondad de ajuste: 
- Chi cuadrado: con este estadístico se puede contrastar la hipótesis nula de que todos los residuos son nulos (Ruiz, 2000). Esta prueba tiene la limitante de que el valor del estadístico de prueba depende del tamaño de muestra, por lo que para muestras grandes, el estadístico tiende a incrementarse.

- Razón de chi-cuadrado entre los grados de libertad $\left(\chi^{2} / g l\right)$ : se utiliza para comparar la magnitud de $\chi^{2}$ respecto a los grados de libertad; para un buen ajuste, esta razón debe ser tan pequeña como sea posible, valores menores a 3 indican ajuste bueno o aceptable (Schermelleh-Engel et al., 2003).

- Índices de ajuste absoluto: evalúan qué tan bien está reproduciendo los datos el modelo propuesto. Existen diferentes índices de ajuste absoluto:

- GFI (goodness of fit index), este índice fue desarrollado por Jöreskog y Sörbom (1984), sus valores son menores o iguales a 1 , un valor de 1 indica un ajuste perfecto.

- $\quad$ AGFI (adjusted goodness of fit index), desarrollado por los mismos autores, corrige el estadístico GFI por los grados de libertad y el número de variables, tanto GFI como AGFI alcanzan el valor de 1 cuando todos los residuos son nulos (Ruiz, 2000).

- RMR (root mean square residual), para este estadístico, los valores pequeños indican mejor ajuste. Un RMR de cero indica ajuste perfecto.

- RMSEA (root mean square error of approximation), valores de .05 o menos indican un buen ajuste del modelo, considerando sus grados de libertad; valores entre .05 y .08 o menos podrían indicar un error razonable de aproximación y valores mayores a .1 indican que el modelo no es adecuado (Browne y Cudeck, 1993).

- Índices de ajuste de incremento: miden el mejoramiento en el ajuste por medio de comparar al modelo propuesto con un modelo en el cual se asume que no hay asociación entre variables observadas y al cual se le suele llamar modelo de independencia. Los siguientes son índices de ajuste de incremento:

- NFI (normed fit index), valores de menos de 9 indican que el modelo puede ser sustancialmente mejorado (Bentler y Bonett, 1980).

- TLI (The Tucker-Lewis coefficient), también conocido como NNFI (non-normed fit index), sus valores se encuentran entre 0 y 1 , valores cercanos a 1 indican muy buen ajuste (op cit.).

- CFI (comparative fit index), valores cercanos a 1 en este estadístico indican muy buen ajuste (Bentler, 1990).
- IFI (incremental fit index), valores cercanos a 1 en este estadístico indican muy buen ajuste (Bollen, 1989).

Las versiones para padres y para hijos del cuestionario se presentan en el Anexo.

Para el segundo objetivo, se trabajó únicamente con los 73 pares (padres y adolescentes de las mismas familias: 73 adultos y 73 jóvenes). Se estimó un puntaje para cada estilo parental, que consiste en la suma de los 15 ítems que forman cada estilo parental. Se valoró el ajuste a la normalidad de ambos estilos parentales, tanto en la muestra de padres como en la muestra de hijos, utilizando la prueba de Kolmogorov-Smirnov, obteniendo valores significativos $(\mathrm{p}<.05)$ en tres de los cuatro puntajes, por lo que se decidió realizar pruebas no paramétricas (prueba de rangos de Wilcoxon) para la comparación de la percepción de estilos parentales entre ambos grupos.

\section{Resultados}

Antes de presentar los resultados de los análisis factoriales y de consistencia interna, se presentan las características de la muestra en la Tabla 1. En la muestra de jóvenes, todos eran solteros, mientras que los padres en su mayoría eran casados (el 83.8\%).

El primer AFE que se realizó fue para valorar una solución de tres factores, buscando la concordancia con los estilos permisivo, autoritativo y autoritario. Se obtuvo un valor de $\mathrm{KMO}=.936$; la varianza explicada fue de $37.4 \%$, los primeros dos factores explicaban el $33.2 \%$ de esta varianza. La distribución de los ítems en los tres factores no coincidía con los ítems que evalúan cada estilo parental. En el tercer factor sólo dos ítems presentaron carga factorial mayor a .40. El AFE se realizó con tres condiciones: (a) Extracción ejes principales, rotación varimax, (b) extracción ejes principales, rotación promax, (c) extracción componentes principales, rotación varimax. El índice de congruencia es bajo $\left(\mathrm{V}_{\min }=.06, \mathrm{~V}_{\max }=.13\right)$, lo que implica que las saturaciones factoriales no se ven afectadas por los métodos utilizados en el AFE.

Enseguida se probó una solución bifactorial que mostró una clara agrupación de los ítems en los estilos autoritario y autoritativo; únicamente cuatro ítems presentaban problema: no se agruparon en el factor correspondiente teóricamente o bien, sus pesos factoriales eran superiores a .40 en ambos factores; estos ítems se eliminaron, al igual que los ítems que teóricamente evalúan el estilo permisivo, debido a que no formaban 
Tabla 1

Características de la muestra.

\begin{tabular}{|c|c|c|c|c|c|}
\hline & & & Edad & $\begin{array}{c}\text { Escolaridad en } \\
\text { años } \\
\end{array}$ & Sexo \\
\hline \multirow{3}{*}{\multicolumn{2}{|c|}{$\begin{array}{l}\text { Padres } \\
\mathrm{n}=197\end{array}$}} & Media & 42.73 & 12.89 & \multirow{3}{*}{$\begin{array}{l}\text { Mujeres }=139(70.6 \%) \\
\text { Hombres }=58(29.4 \%)\end{array}$} \\
\hline & & Mediana & 42.00 & 13.00 & \\
\hline & & Desviación típica & 7.47 & 3.61 & \\
\hline \multirow{3}{*}{\multicolumn{2}{|c|}{$\begin{array}{l}\text { Hijos } \\
n=307\end{array}$}} & Media & 15.92 & 10.75 & \multirow{3}{*}{$\begin{array}{l}\text { Mujeres }=192(62.5 \%) \\
\text { Hombres }=115(37.5 \%)\end{array}$} \\
\hline & & Mediana & 16.00 & 11.00 & \\
\hline & & Desviación típica & 1.57 & 2.03 & \\
\hline \multirow{6}{*}{ 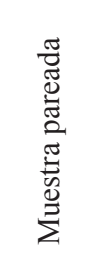 } & & Media & 42.70 & 11.97 & \multirow{3}{*}{$\begin{array}{l}\text { Mujeres }=53(72.6 \%) \\
\text { Hombres }=20(27.4 \%)\end{array}$} \\
\hline & Padres & Mediana & 42.00 & 12.00 & \\
\hline & $\mathrm{n}=73$ & Desviación típica & 6.27 & 3.69 & \\
\hline & & Media & 15.15 & 10.11 & \multirow{3}{*}{$\begin{array}{l}\text { Mujeres }=42(57.7 \%) \\
\text { Hombres }=31(42.5 \%)\end{array}$} \\
\hline & Hijos & Mediana & 15.00 & 11.00 & \\
\hline & $\mathrm{N}=73$ & Desviación típica & 1.30 & 1.77 & \\
\hline
\end{tabular}

un sólo factor. Posteriormente se estimó de nuevo el AFE, confirmando la distribución de ítems en esos dos factores. Finalmente, se seleccionaron los 15 ítems para cada factor, que presentaron valores más altos en la correlación inter-ítem y en las cargas factoriales.

El AFE, con el método de ejes principales y rotación varimax de esta versión reducida de 2 factores de 15 ítems cada uno, se presenta en la tabla 2. Al comparar las saturaciones en las tres condiciones utilizadas para el AFE, el índice de congruencia es bajo $\left(\mathrm{V}_{\min }=.06\right.$, $\mathrm{V}_{\max }=.08$ ), lo que implica que las saturaciones factoriales no se ven afectadas por los métodos utilizados en el AFE. El valor de KMO fue de .935 y la varianza explicada por los dos factores fue de $44.1 \%$. Asimismo, se confirmó la estructura factorial y se analizó la consistencia interna en las muestras de padres e hijos por separados (ver Tabla 2).

Para concluir con el análisis de las propiedades psicométricas del cuestionario en su versión breve, se realizó un Análisis Factorial Confirmatorio con la muestra completa $(\mathrm{n}=504)$. Todos los parámetros fueron significativos, sin embargo, los estadísticos de bondad de ajuste indican que el modelo puede ser mejorado (Tabla 3 ), esto se toma en cuenta en las limitaciones del estudio.

Como se mencionó en el procedimiento para el segundo objetivo, se trabajó únicamente con los 73 pares (padres y adolescentes de las mismas familias). En la tabla 4 se muestran las medidas de tendencia central para los dos estilos parentales, medidos con la versión breve del cuestionario. Puede observarse que los padres se perciben más autoritativos de lo que los perciben sus hijos, lo que se confirma al realizar el contraste con la prueba de rangos de Wilcoxon $(\mathrm{Z}=-3.182 ; \mathrm{p}=.001)$. Por el contrario, para los puntajes de estilo autoritario los valores son muy similares entre el grupo de padres y el grupo de hijos, pues no se encontró una diferencia significativa entre los grupos $(\mathrm{Z}=-.062 ; \mathrm{p}=.951)$.

Finalmente, se elaboraron gráficas donde se reflejan los puntajes de cada uno de los 73 pares en ambos estilos parentales, se ordenaron los puntajes de menor

Figura 1.

Análisis Factorial Confirmatorio del Cuestionario Breve de Prácticas Parentales.

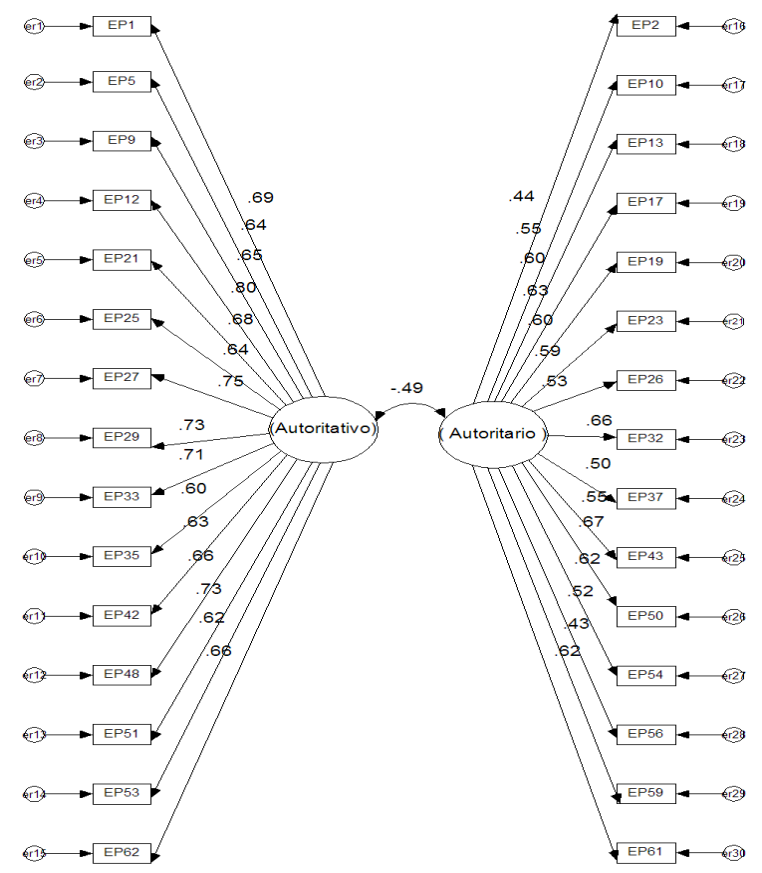


Tabla 2.

Cargas factoriales para el Cuestionario Breve de Prácticas Parentales.

\begin{tabular}{|c|c|c|c|c|c|c|}
\hline \multirow[b]{2}{*}{ Ítems } & \multicolumn{2}{|c|}{ Muestra completa $n=504$} & \multicolumn{2}{|c|}{$\underline{\text { Muestra de padres } n=197}$} & \multicolumn{2}{|c|}{$\underline{\text { Muestra de hijos } n=307}$} \\
\hline & $\begin{array}{c}\text { Autoritativo } \\
24.9 \% \text { de varianza } \\
\text { Alfa }=.93\end{array}$ & $\begin{array}{c}\text { Autoritario } \\
\begin{array}{c}19.1 \% \text { de } \\
\text { varianza } \\
\text { Alfa }=.88\end{array}\end{array}$ & $\begin{array}{c}\text { Autoritativo } \\
21.2 \% \text { de } \\
\text { varianza } \\
\text { Alfa }=.89\end{array}$ & $\begin{array}{c}\text { Autoritario } \\
\begin{array}{c}19.1 \% \text { de } \\
\text { varianza } \\
\text { Alfa }=.88\end{array}\end{array}$ & $\begin{array}{c}\text { Autoritativo } \\
\begin{array}{c}25.8 \% \text { de } \\
\text { varianza } \\
\text { Alfa }=.93\end{array}\end{array}$ & $\begin{array}{c}\text { Autoritario } 19.3 \% \\
\text { de varianza } \\
\text { Alfa }=.88\end{array}$ \\
\hline 1 & .672 & & .460 & & .736 & \\
\hline 5 & .622 & & .456 & & .638 & \\
\hline 9 & .594 & & .398 & & .638 & \\
\hline 12 & .763 & & .576 & & .784 & \\
\hline 21 & .620 & & .607 & & .622 & \\
\hline 25 & .654 & & .654 & & .661 & \\
\hline 27 & .718 & & .701 & & .697 & \\
\hline 29 & .716 & & .695 & & .708 & \\
\hline 33 & .667 & & .546 & & .683 & \\
\hline 35 & .595 & & .498 & & .606 & \\
\hline 42 & .617 & & .634 & & .616 & \\
\hline 48 & .620 & & .604 & & .628 & \\
\hline 51 & .693 & & .514 & & .747 & \\
\hline 53 & .650 & & .608 & & .650 & \\
\hline 62 & .686 & & 649 & & .692 & \\
\hline 2 & & .453 & & .415 & & .468 \\
\hline 10 & & .526 & & .511 & & .533 \\
\hline 13 & & .588 & & .634 & & .579 \\
\hline 17 & & .582 & & .638 & & .564 \\
\hline 19 & & .615 & & .570 & & .630 \\
\hline 23 & & .566 & & .643 & & .542 \\
\hline 26 & & .454 & & .423 & & .457 \\
\hline 32 & & .631 & & .726 & & .587 \\
\hline 37 & & .512 & & .395 & & .560 \\
\hline 43 & & .525 & & .440 & & .557 \\
\hline 50 & & .632 & & .655 & & .620 \\
\hline 54 & & .601 & & .485 & & .656 \\
\hline 56 & & .492 & & .499 & & .491 \\
\hline 59 & & .457 & & .456 & & .480 \\
\hline 61 & & .619 & & .618 & & .589 \\
\hline
\end{tabular}

Nota: se excluyen las cargas factoriales menores a .39 para facilitar la interpretación.

Tabla 3.

Índices de bondad de ajuste para el Análisis Factorial Confirmatorio.

\begin{tabular}{lcccccccccc}
\hline & & & \multicolumn{3}{c}{ Índices de ajuste absoluto } & \multicolumn{3}{c}{ Índices de ajuste de incremento } \\
\cline { 6 - 12 } $\mathrm{X}^{2}$ & g.1. & $\chi^{2} / \mathrm{gl}$ & GFI & AGFI & RMR & RMSEA & NFI & TLI & CFI & IFI \\
\hline 1258.8 & 404 & 3.116 & .843 & .820 & .071 & .065 & .814 & .855 & .865 & .866 \\
\hline
\end{tabular}


Tabla 4

Medidas de tendencia central para los estilos parentales en la muestra pareada.

\begin{tabular}{llcc}
\hline Grupo & & Autoritativo & Autoritario \\
\hline \multirow{3}{*}{ Padres $(\mathrm{n}=73)$} & Media & 61.53 & 28.42 \\
& Mediana & 65.00 & 27.00 \\
& Desviación típica & 10.76 & 8.29 \\
\multirow{2}{*}{ Hijos $(\mathrm{n}=73)$} & Media & 54.97 & 28.85 \\
& Mediana & 57.00 & 27.00 \\
& Desviación típica & 13.38 & 8.08 \\
\hline
\end{tabular}

Figura 2.

Puntajes de cada par (padres e hijos) para ambos estilos parentales.
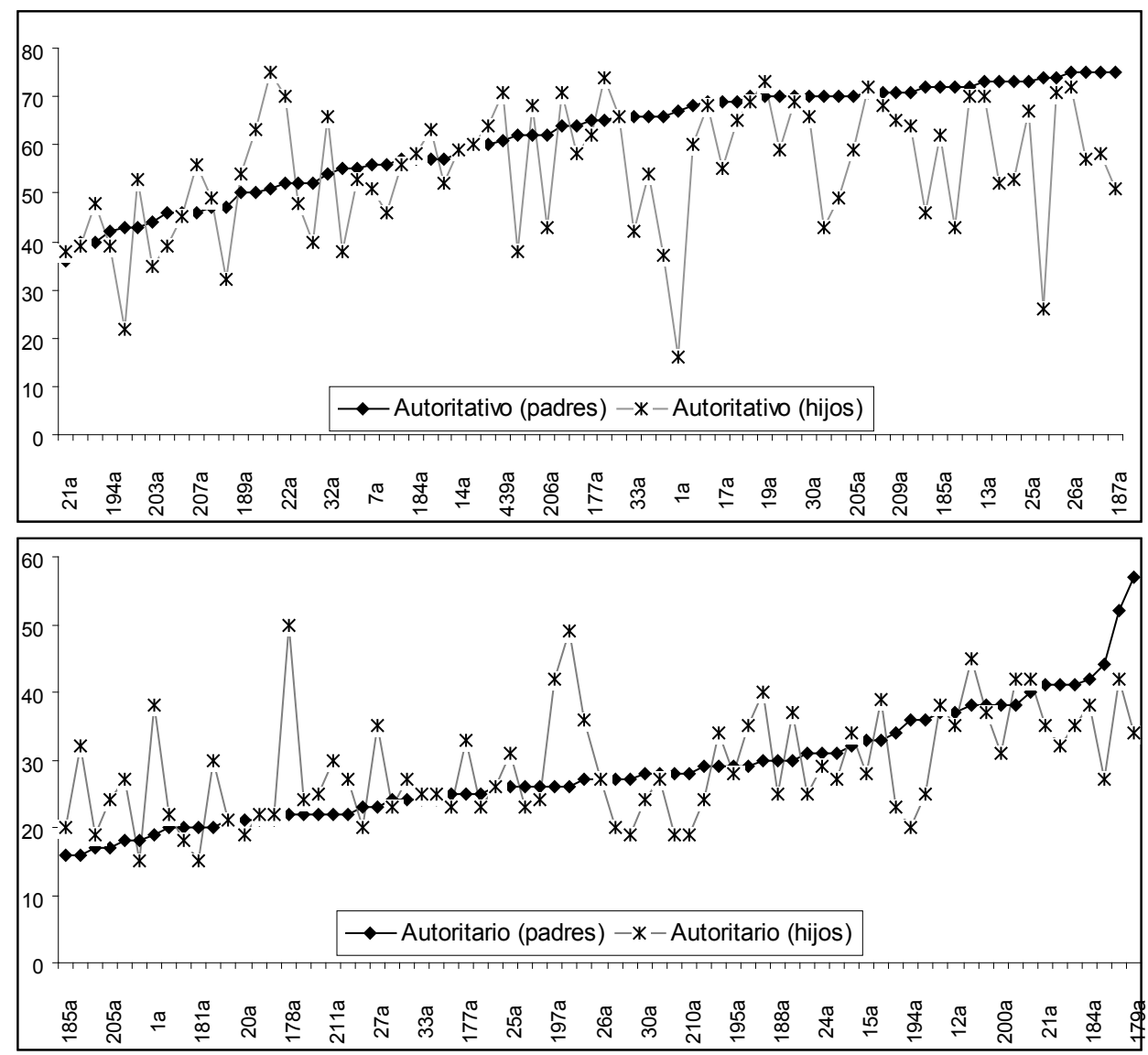

a mayor para el grupo de padres y se presenta el valor correspondiente al adolescente de la misma familia, estas gráficas se exiben en la figura 2. Puede observarse que los puntajes más bajos son de los adolescentes, quienes señalan que sus padres son menos autoritativos de lo que ellos se consideran, asimismo, aunque existen coincidencias, hay familias en las que dista mucho la percepción de los padres de la percepción de los hijos. Por otro lado, en lo que respecta al estilo autoritario, cabe resaltar que en las familias donde los padres se consideran más autoritarios, los adolescentes no alcanzaron puntajes tan altos. 


\section{Discusión}

La medición por autoinforme conlleva un sesgo que se ha aceptado en las investigaciones sociales por las ventajas en su utilización; sin embargo, es necesario trabajar con instrumentos que reflejen adecuadas propiedades de confiabilidad y validez. En el caso que nos compete, se presentan resultados no muy alentadores respecto al cuestionario. Tomando las subescalas propuestas por los autores de la escala, la consistencia interna es inadecuada en varias de ellas. Por otro lado, la versión breve propuesta en esta investigación cuenta con consistencia interna adecuada y el AFE converge al dividir la muestra entre padres e hijos; aun así, el modelo estimado para el AFC es susceptible de ser mejorado ya que los índices de bondad de ajuste no son adecuados. Esto es una limitación importante en los resultados encontrados.

El tratarse de un instrumento traducido y no construido para la cultura mexicana, puede ser una explicación de los resultados anteriores; Robinson et al. (1996) documentaron las diferencias en estilos parentales al comparar cuatro culturas (Australia, Rusia, China y Estados Unidos), mientras que Robles y Oudhf Van Barneveld (2010) indicaron que la familia mexicana tiene características distintivas, similar a las culturas latinas donde la vida familiar suele ser más flexible y menos planificada y formal; es así que su estudio reflejó que las prácticas de crianza que realizan las madres procedentes de una ciudad del centro de México, tienen muchos aspectos que coinciden con las características de los procesos de socialización familiar en otros contextos socioculturales, pero que también existen ciertas particularidades propias relacionadas con los rasgos específicos de la familia mexicana.

En cuanto a la diferencia entre grupos, los resultados de la investigación muestran que los estilos parentales reportados por padres e hijos no coinciden totalmente, especialmente en el estilo autoritativo, donde los padres se consideran más autoritativos de lo que los consideran sus hijos.

Lo anterior concuerda con lo encontrado por Smetana (1995), quien comparó la percepción de los estilos parentales de los padres y sus hijos adolescentes, cuyos resultados indican que los adolescentes percibieron a sus padres más permisivos y más autoritarios, mientras que los padres se percibieron a ellos mismos más autoritativos. Por otro lado, Bersabé, Fuentes y Motrico (2001) encontraron una baja concordancia entre la información aportada por los padres y sus hijos.

La diferencia hallada entre lo reportado por padres e hijos pudiera deberse a las expectativas de padres e hijos con respecto a los comportamientos apropiados; los padres suelen valorar más la ausencia de problemas comportamentales y los valores convencionales como obedecer las normas, mientras que los adolescentes prefieren aquellos valores relacionados con la sociabilidad y la autonomía personal (Collins, 1997, en Oliva y Parra, 2004).

Las respuestas de los padres están basadas en el conocimiento que éstos tienen sobre el comportamiento de sus hijos y pueden estar sesgadas a veces por la deseabilidad social. En el caso de adolescentes, son ellos mismos quienes proporcionan la información sobre el estilo de sus padres, y de acuerdo a Gonzáles, Cauce y Mason (1996, en Oliva, Parra, Sánchez-Queija y López, 2007), esta percepción suele mostrar menos sesgos y resultar más objetiva.

Por otro lado, Oliva, Parra y Arranz (2008) señalan que los escasos estudios que han evaluado los estilos de padre y madre de forma separada, han encontrado que a pesar de que la coincidencia entre los progenitores es elevada, dista de ser total, por ejemplo, Baumrind (1991) encontró que un 76\% de parejas coincidía en su estilo. Estos análisis no se realizaron en el presente estudio, dado que sólo se evaluó a uno de los progenitores de cada familia y por otro lado, era mayor la muestra de madres. Resulta necesario que este tema se considere en futuras investigaciones.

Con respecto a las consecuencias que la falta de coherencia puede tener para el ajuste adolescente, Fletcher, Steinberg y Sellers (1999) hallaron que los adolescentes que tenían sólo un padre democrático mostraban una mayor competencia que aquellos que no tenían ninguno, aunque padre y madre fueran consistentes en sus estilos. Por lo tanto, aunque la inconsistencia disciplinaria ha sido considerada como una fuente de estrés para los padres, con repercusiones negativas en los hijos, los beneficios de disponer al menos de un progenitor democrático parecen superar los efectos negativos que se podrían derivar de la falta de acuerdo.

Sin embargo, es necesario que los padres eduquen de manera interdependiente a sus hijos, y que ambos utilicen el mismo estilo, lo que facilitaría los acuerdos entre ellos e impactaría en la relación matrimonial, que es un aspecto clave en el desarrollo de los hijos (Winsler, Madigan y Aquilino, 2005).

Finalmente, los resultados mencionados obligan a preguntarse si las ventajas que ha reportado la literatura en el estilo autoritativo se confirmarían al evaluar el estilo parental a través de la percepción de los hijos (o un observador imparcial). Entre las ventajas que se han reportado podemos mencionar la correlación con la creatividad y los niveles de frustración, el compromiso efectivo con el estudio y las estrategias de aprendizaje, el 
buen desempeño académico y la madurez psicológica, la motivación intrínseca, autonomía, capacidad de relación y autorregulación del comportamiento y emociones y la auto-actualización en estudiantes universitarios (Bojeva, 1998; Cristian y Snowden, 1999; Domínguez y Carton, 1997; Dorrnsbusch, Ritter, Leiderman, Roberts, y Fraleigh, 1987; Grolnick y Kurowski, 1999).

\section{Referencias}

Baumrind, D. (1971). Current patterns of parental authority. Developmental Psychology, 4 (1, Pt. 2), 1-103.

Baumrind, D. (1991). The influence of parenting style on adolescent competence and substance use. Journal of Early Adolescence, 11 (1), 56-95.

Bersabé, R., Fuentes M. J. y Motrico, E. (2001). Análisis psicométrico de dos escalas para evaluar estilos educativos parentales. Psicothema, 13 (4), 678-684.

Bojeva, M. (1998). Parenting styles and adolescents' learning strategies in the urban community. Journal of Multicultural Counseling and Development, 62, 110-119.

Browne, M. y Cudeck, R. (1993). Alternative ways of assessing model fit. En K.A. Bollen y J.S. Long (Eds.), Testing structural equation models. Newbury Park, CA: Sage, 136-162.

Buri, J. R. (1989). Self-esteem and Appraisals of Parental Behavior. Adolescent Research, 4 (1), 33-49.

Buri, J. R. (1991). Parental Authority Questionnaire, Journal of Personality and Social Assessment, 57 (1), 110-119.

Coloma, M. (1993). Estilos educativos paternos. En Quintana, C. (coord.). Pedagogía familiar (pp. 45-58). Madrid: Narcea.

Cristian, L. \& Snowden, P. (1999). Parenting the young gifted child: Supportive behaviors. Roeper Review, 21, 215-221.

Darling, N. \& Steinberg, L. (1993). Parenting Style as Context: An integrative Model. Psychological Bulletin, 113 (3), 487-496.

Domínguez, M. y Carton, J. (1997). The relationship between selfactualization and parenting style. Journal of Social Behavior \& Personality, 12 (4), 1093-1101.

Dornbush, S., Ritter, P., Leiderman, H., Roberts, D. \& Fraleigh, M. (1987). The relation of parenting style to adolescent school performance. Child Development, 58, 1244-1257.

Fletcher, A., Steinberg, L. \& Sellers, E. (1999). Adolescents' WellBeing as a Function of Perceived Interparental Consistency. Journal of Marriage and the Family, 61, 599-610.

Gaxiola, J., Frías, M., Cuamba, N., Franco, J. y Olivas, L. (2006). Validación del cuestionario de prácticas parentales en una población mexicana. Enseñanza e investigación en Psicología, 11 (1), 115-128.

Grolnick, W. \& Kurowski, C. (1999). Family processes and the development of children's self-regulation. Educational Psychologist, 34 (1), 3-14.

Grusec, J. E., Goodnow, J. J. \& Kuczynksi, L. (2000). New directions in analyses of parenting contributions to children's acquisition of values. Child Development. 71 (1), 205-211.

Holmbeck, G.N., Paikoff, R. \& Brooks-Gunn, J. (1995). Parenting adolescents. In M. Bornstein (Ed.), Handbook of parenting: Vol. 1. Children and parenting (pp. 91-118), Mahwah, NJ: Erlbaum. Huver, R., Otten, R., de Vries, H. y Engels, R. (2010). Personality and parenting style in parents of adolescents. Journal of Adolescence, 33 (3), 395-402.
Lee, S. M., Daniels, M. H. \& Kissinger, D. B. (2006). Parental Influences on dolescent Adjustment: Parenting Styles Versus Parenting Practices. Family Journal: Counseling and Therapy for Couples and Families, 14 (3), 253-259.

Maccoby, E. E. \& Martin, J. A. (1983). Socialization in the context of the family: Parent-child interaction. En P. H. Mussen \& E. M. Hetherington (Ed.). Handbook of child psychology: Vol. 4. Socialization, personality, and social development (4th ed.). New York: Wiley.

MacCallum, R. C., Widaman, K. F., Zhang, S. y Hong, S. (1999). Sample Size in Factor Analysis. Psychological Methods, 4 (1), 84-99.

Marsh, H. W. \& Hocevar, D. (1985). Application of confirmatory factor analysis to the study of self-concept: First-and higher-order factor models and their invariance across groups. Psychological Bulletin, 97, 562-582.

Medina, R. (2004). Contextos y circunstancias de la nueva paternidad en busca del instinto paterno: implicaciones para la terapia familiar. Revista Universidad de Guadalajara, (30). Disponible en: http://www.cge.udg.mx/revistaudg/rug30/opinion2.html.

Oliva, A. y Parra, J. (2004). Contexto familiar y desarrollo psicológico durante la adolescencia. En E. Arranz (Ed.). Familia y desarrollo psicológico (pp. 96-123), Madrid: Pearson Educación.

Oliva, A., Parra Á., Sánchez-Queija, I. y López, F. (2007). Estilos educativos materno y paterno: Evaluación y relación con el ajuste adolescente. Anales de psicología, 23 (1), 49-56.

Oliva, A., Parra, A. y Arranz, E. (2008). Estilos relacionales parentales y ajuste adolescente. Infancia y Aprendizaje, 31 (1), 93-106.

Osorio de Rebellón, A., Rivas, S., Jokin de Irala, M. y López, C. (2009). Evaluación de los estilos educativos parentales en una muestra de estudiantes filipinos: implicaciones educativas. Revista Panamericana de Pedagogía, 14, 13-37.

Robinson, C. C., Mandelco, B., Frost, O. S. \& Hart, C. H. (1995). Authoritative, authoritarian, and permissive parenting practices: Development of a new measure. Psychological Reports, 77, 819-830.

Robinson, C. C., Hart, C. H., Mandelco, B., Olsen S., Russel, A., Aloa, V., Jin, S., Nelson, D. \& Bazarskaya, N. (1996, agosto). Psychometric support for a new measure of authoritative, authoritarian, and permissive parenting practices: cross-cultural connections. Conference presented in 14th Biennial International Society for the study of Behavioral Development, Quebec, Canadá, 12-16. Disponible en: http://www.eric.ed.gov/ERICDocs/data/ericdocs2sq1/content_storage_01/0000019b/80/14/c3/f5.pdf

Robles, E. \& Oudhf Van Barneveld, H. (2010). Validación de un cuestionario de tareas de crianza en mujeres mexicanas. Pensamiento Psicológico, 7 (14), 73-80.

Ruiz, M. (2000). Introducción a los modelos de ecuaciones estructurales. Madrid: Ediciones UNED.

Smetana, J. G. (1995). Parenting styles and conceptions of parental authority during adolescence, Child Development., 66 (2), 299316.

Spera, C. (2005). A Review of the Relationship among Parenting Practices, Parenting Styles, and Adolescent School Achievement. Educational Psychology Review, 17 (2), 125-146.

Winsler, A., Madigan, A. y Aquilino, S. (2005). Correspondence between maternal and paternal parenting styles in early childhood. Early Childhood Research Quarterly 20 (1), 1-12.

Yárnoz, S. Y. (2006). ¿Seguimos descuidando a los padres? El papel del padre en la dinámica familiar y su influencia en el bienestar psíquico de sus componentes. Anales de psicología, 22 (2), 175-185. 
ANEXOS

Cuestionario Breve de Prácticas Parentales (versión para padres)

\begin{tabular}{|c|c|c|c|c|c|}
\hline Marque la opción que se adecue a su situación: & $\stackrel{\text { }}{\Xi}$ & $\begin{array}{l}0 \\
0 \\
0 \\
D \\
0 \\
0 \\
0 \\
0 \\
0\end{array}$ & 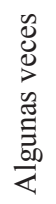 & 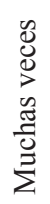 & 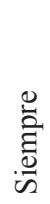 \\
\hline EP1. Motivo a mi hijo(a) a hablar de sus problemas & 1 & 2 & 3 & 4 & 5 \\
\hline EP2. Disciplino a mi hijo(a) por medio del castigo más que usando la razón & 1 & 2 & 3 & 4 & 5 \\
\hline EP5. Felicito a mi hijo(a) cuando se porta bien & 1 & 2 & 3 & 4 & 5 \\
\hline EP9. Muestro comprensión (empatía) cuando mi hijo(a) se encuentra herido(a) o frustrado(a) & 1 & 2 & 3 & 4 & 5 \\
\hline EP10. Castigo a mi hijo(a) quitándole privilegios con poca o ninguna explicación & 1 & 2 & 3 & 4 & 5 \\
\hline EP12. Brindo confianza y entendimiento cuando mi hijo(a) se encuentra triste & 1 & 2 & 3 & 4 & 5 \\
\hline EP13. Grito y rezongo cuando mi hijo(a) se porta mal & 1 & 2 & 3 & 4 & 5 \\
\hline EP17. Ofendo y critico para que mi hijo(a) mejore & 1 & 2 & 3 & 4 & 5 \\
\hline EP19. Jalo con fuerza a mi hijo(a) cuando es desobediente & 1 & 2 & 3 & 4 & 5 \\
\hline EP21. Estoy atenta(o) a los deseos y necesidades de mi hijo(a) & 1 & 2 & 3 & 4 & 5 \\
\hline EP23. Discuto con mi hijo(a) & 1 & 2 & 3 & 4 & 5 \\
\hline EP25. Explico a mi hijo(a) las razones de las reglas que deben ser obedecidas & 1 & 2 & 3 & 4 & 5 \\
\hline EP26. Me preocupo más de mis sentimientos que de los sentimientos de mis hijo(a)s & 1 & 2 & 3 & 4 & 5 \\
\hline EP27. Le digo a mi hijo(a) que aprecio sus logros o sus intentos de lograr algo & 1 & 2 & 3 & 4 & 5 \\
\hline $\begin{array}{l}\text { EP29. Ayudo a mi hijo(a) a entender el impacto de la conducta motivándolo(a) a que hable acerca de las } \\
\text { consecuencias de sus propias acciones }\end{array}$ & 1 & 2 & 3 & 4 & 5 \\
\hline EP32. Exploto en enojo con mi hijo(a) & 1 & 2 & 3 & 4 & 5 \\
\hline EP33. Me doy cuenta de los problemas y preocupaciones que tiene mi hijo(a) en la escuela & 1 & 2 & 3 & 4 & 5 \\
\hline EP35. Le expreso afecto a mi hijo(a) con abrazos o besos (o al cargarlo) & 1 & 2 & 3 & 4 & 5 \\
\hline EP37. Empleo el castigo físico como una manera de disciplinar a mi hijo(a) & 1 & 2 & 3 & 4 & 5 \\
\hline EP42. Hablo y razono con mi hijo(a) cuando se porta mal & 1 & 2 & 3 & 4 & 5 \\
\hline EP43. Doy una cachetada a mi hijo(a) cuando se porta mal & 1 & 2 & 3 & 4 & 5 \\
\hline EP48. Animo a mi hijo(a) a que libremente exprese lo que siente cuando no está de acuerdo conmigo & 1 & 2 & 3 & 4 & 5 \\
\hline EP50. Ofendo y critico a mi hijo(a) cuando no hace bien lo que tiene que hacer & 1 & 2 & 3 & 4 & 5 \\
\hline EP51. Respeto las opiniones de mi hijo(a) al ayudarle a que las exprese & 1 & 2 & 3 & 4 & 5 \\
\hline EP53. Le explico a mi hijo(a) cómo me siento con su buena o mala conducta & 1 & 2 & 3 & 4 & 5 \\
\hline EP54. Amenazo a mi hijo(a) con castigarlo(a) con poca o ninguna justificación & 1 & 2 & 3 & 4 & 5 \\
\hline $\begin{array}{l}\text { EP56. Cuando mi hijo(a) me pregunta por qué tiene que hacer algo, le contesto que porque yo lo digo o } \\
\text { porque soy su mamá (o papá) o porque así lo quiero }\end{array}$ & 1 & 2 & 3 & 4 & 5 \\
\hline EP59. Exijo (demando) a mi hijo(a) que haga las cosas & 1 & 2 & 3 & 4 & 5 \\
\hline EP61. Jalo (o jaloneo) a mi hijo(a) cuando es desobediente & 1 & 2 & 3 & 4 & 5 \\
\hline EP62. Explico a mi hijo(a) las razones de las reglas (de la casa) & 1 & 2 & 3 & 4 & 5 \\
\hline
\end{tabular}


Cuestionario Breve de Prácticas Parentales (versión para hijos)

Marca la opción que mejor se adecue a tu situación:

EP1. Mis padres me motivan a hablar con ellos de mis problemas

$\begin{array}{lllll}1 & 2 & 3 & 4 & 5\end{array}$

EP2. Mis padres me disciplinan por medio del castigo más que usando la razón

EP5. Mis padres me felicitan cuando me porto bien

EP9. Mis padres muestran comprensión (empatía) cuando me encuentro herido(a) o frustrado(a)

$\begin{array}{lllll}1 & 2 & 3 & 4 & 5\end{array}$

EP10. Mis padres me castigan quitándome privilegios con poca o ninguna explicación

$\begin{array}{lllll}1 & 2 & 3 & 4 & 5\end{array}$

EP12. Mis padres me brindan confianza y entendimiento cuando me encuentro triste

$\begin{array}{lllll}1 & 2 & 3 & 4 & 5\end{array}$

EP13. Mis padres me gritan o rezongan cuando me porto mal

EP17. Mis padres me ofenden y critican para que mejore

EP19. Mis padres me jalan (o jalonean) con fuerza cuando soy desobediente

EP21. Mis padres están atentos a mis deseos y necesidades

$\begin{array}{lllll}1 & 2 & 3 & 4 & 5\end{array}$

$\begin{array}{lllll}1 & 2 & 3 & 4 & 5\end{array}$

EP23. Mis padres discuten conmigo

EP25. Mis padres me explican las razones de las reglas que deben ser obedecidas

EP26. Mis padres se preocupan más de sus sentimientos que de los míos

EP27. Mis padres me dicen que aprecian mis logros o intentos de lograr algo

EP29. Mis padres me motivan a que hable acerca de las consecuencias de mis propias acciones

$\begin{array}{lllll}1 & 2 & 3 & 4 & 5\end{array}$

$\begin{array}{lllll}1 & 2 & 3 & 4 & 5\end{array}$

$\begin{array}{lllll}1 & 2 & 3 & 4 & 5\end{array}$

$\begin{array}{lllll}1 & 2 & 3 & 4 & 5\end{array}$

EP32. Mis padres explotan en enojo conmigo

EP33. Mis padres se dan cuenta de mis problemas y preocupaciones de la escuela (o trabajo)

$\begin{array}{lllll}1 & 2 & 3 & 4 & 5\end{array}$

$\begin{array}{lllll}1 & 2 & 3 & 4 & 5\end{array}$

$\begin{array}{lllll}1 & 2 & 3 & 4 & 5\end{array}$

$\begin{array}{lllll}1 & 2 & 3 & 4 & 5\end{array}$

$\begin{array}{lllll}1 & 2 & 3 & 4 & 5\end{array}$

EP35. Mis padres me expresan afecto con abrazos o besos

$\begin{array}{lllll}1 & 2 & 3 & 4 & 5\end{array}$

EP37. Mis padres emplean el castigo físico como una manera de disciplinarme

$\begin{array}{lllll}1 & 2 & 3 & 4 & 5\end{array}$

EP42. Mis padres hablan y razonan conmigo cuando me porto mal

$\begin{array}{lllll}1 & 2 & 3 & 4 & 5\end{array}$

EP43. Mis padres me dan una cachetada cuando me porto mal

$\begin{array}{lllll}1 & 2 & 3 & 4 & 5\end{array}$

EP48. Mis padres me animan a que libremente exprese lo que siento cuando no estoy de acuerdo con ellos

$\begin{array}{lllll}1 & 2 & 3 & 4 & 5\end{array}$

EP50. Mis padres me ofenden y critican cuando no hago bien lo que tengo que hacer

$\begin{array}{lllll}1 & 2 & 3 & 4 & 5\end{array}$

EP51. Mis padres respetan mis opiniones ayudándome a que las exprese

$\begin{array}{lllll}1 & 2 & 3 & 4 & 5\end{array}$

EP53. Mis padres me explican cómo se sienten con mi buena o mala conducta

$\begin{array}{lllll}1 & 2 & 3 & 4 & 5\end{array}$

EP54. Mis padres me amenazan con castigarme con poca o ninguna justificación

$\begin{array}{lllll}1 & 2 & 3 & 4 & 5\end{array}$

EP56. Cuando pregunto a mis padres por qué tengo que hacer algo, me contestan que porque ellos lo dicen o porque es mi mamá (o papá) o porque así lo quieren

$\begin{array}{lllll}1 & 2 & 3 & 4 & 5\end{array}$

EP59. Mis padres me exigen que haga las cosas

$\begin{array}{lllll}1 & 2 & 3 & 4 & 5\end{array}$

EP61. Mis padres me jalan (jalonean) cuando soy desobediente

$\begin{array}{lllll}1 & 2 & 3 & 4 & 5\end{array}$

EP62. Mis padres me explican las razones de las reglas (de la casa)

$\begin{array}{lllll}1 & 2 & 3 & 4 & 5\end{array}$

$\begin{array}{lllll}1 & 2 & 3 & 4 & 5\end{array}$ 